E R R A T A

RELATIVISTIC SCATTERING OF TWO EQUALMASS PARTICLES. William B. Rolnick and R. M. Thaler [Phys. Rev. Letters 14, 572 (1965)], and

RELATIVISTIC SMALL-ANGLE SCATTERING OF TWO CHARGED PARTICLES. William B. Rolnick and R. M. Thaler [Phys. Rev. Letters 15, 117 (1965)].

Our use of the potential $Z_{1} Z_{2} e^{2} / r_{12}$ in the center-of-momentum system yields a forward-scattering cross section which does not agree with the one-photon-exchange result at relativistic velocities. Thus, the calculation we proposed would have to be modified through the use of $N Z_{1} Z_{2} e^{2} / r_{12}$ for the longest range part of the equivalent electromagnetic Bakamjiam-Thomas potential. The value of $N$ which is required to yield the same forward-scattering cross section as the one-photon exchange cross section is $N$ $=1+k^{2} c^{2} / E_{1} E_{2}=1-\overrightarrow{\mathrm{v}}_{1} \cdot \overrightarrow{\mathrm{v}}_{2} / c^{2}$, where $\overrightarrow{\mathrm{v}}_{i}$ is the velocity of particle $i$ and $k$ is the magnitude of the momentum of either particle in the center-ofmomentum system. The term $Z_{1} Z_{2} e^{2} \vec{v}_{1} \cdot \vec{v}_{2} / c^{2} r$ in the interaction may be interpreted as the current-current contribution, which at relativistic velocities is of the same order as the static part.
The corrected phase of the small-angle scattering amplitude as calculated by us is then

$$
2\left(\sigma_{0}-\eta \ln \frac{1}{2} \theta_{\text {c.m. }}\right) \text {, }
$$

where $\theta_{\text {c.m. }}$ is the center-of-momentum scattering angle with

$$
\eta=\left(1-\overrightarrow{\mathrm{v}}_{1} \cdot \overrightarrow{\mathrm{v}}_{2} / c^{2}\right) Z_{1} Z_{2} e^{2} / \hbar\left|\overrightarrow{\mathrm{v}}_{1}-\overrightarrow{\mathrm{v}}_{2}\right|,
$$

and

$$
\sigma_{0}=\arg \Gamma(1+i \eta)
$$

In the ultrarelativistic limit

$$
\eta \rightarrow Z_{1} Z_{2} e^{2} / \hbar c \text {. }
$$

We are indebted to Dr. John S. Bell for pointing out to us that our result could not be in agreement with the one-photon-exchange cross-section.

\section{ELECTRODYNAMICS OF INTERMEDIATE} BOSONS AND CP NONINVARIANCE. Tai Tsun $\mathrm{Wu}$ [Phys. Rev. Letters 15, 718 (1965)].

The sentence after Eq. (14) should read as follows: "The additional term is invariant under $C$ but not $P$ or $T . "$ 